\title{
Investigation into the Role of Initial Conditions on Rayleigh-Taylor Instabilities by Low Atwood Experiments and Simulations
}

\author{
N. Mueschke, M. Andrews, and O. Schilling
}

July 16, 2003

U.S. Department of Energy

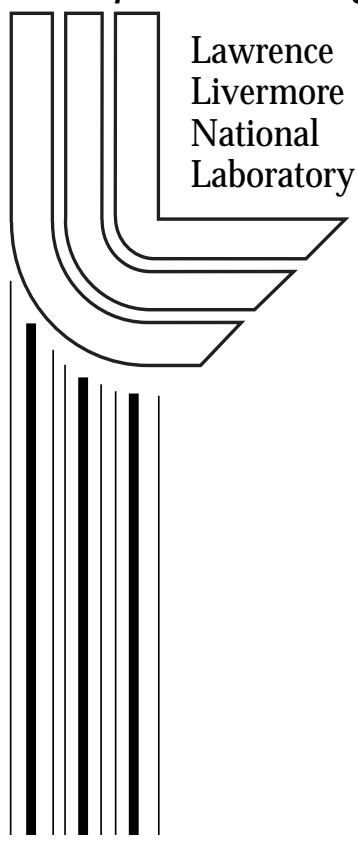




\section{DISCLAIMER}

This document was prepared as an account of work sponsored by an agency of the United States Government. Neither the United States Government nor the University of California nor any of their employees, makes any warranty, express or implied, or assumes any legal liability or responsibility for the accuracy, completeness, or usefulness of any information, apparatus, product, or process disclosed, or represents that its use would not infringe privately owned rights. Reference herein to any specific commercial product, process, or service by trade name, trademark, manufacturer, or otherwise, does not necessarily constitute or imply its endorsement, recommendation, or favoring by the United States Government or the University of California. The views and opinions of authors expressed herein do not necessarily state or reflect those of the United States Government or the University of California, and shall not be used for advertising or product endorsement purposes.

This work was performed under the auspices of the U. S. Department of Energy by the University of California, Lawrence Livermore National Laboratory under Contract No. W-7405-Eng-48.

This report has been reproduced directly from the best available copy.

Available electronically at http://www.doc.gov/bridge

Available for a processing fee to U.S. Department of Energy

And its contractors in paper from

U.S. Department of Energy

Office of Scientific and Technical Information

P.O. Box 62

Oak Ridge, TN 37831-0062

Telephone: (865) 576-8401

Facsimile: (865) 576-5728

E-mail: reports@adonis.osti.gov

Available for the sale to the public from

U.S. Department of Commerce

National Technical Information Service

5285 Port Royal Road

Springfield, VA 22161

Telephone: (800) 553-6847

Facsimile: (703) 605-6900

E-mail: orders@ntis.fedworld.gov

Online ordering: http://www.ntis.gov/ordering.htm

\section{OR}

Lawrence Livermore National Laboratory

Technical Information Department's Digital Library

http://www.llnl.gov/tid/Library.html 


\title{
RESEARCH HIGHLIGHT \\ Investigation into the Role of Initial Conditions on Rayleigh-Taylor Instabilities by Low Atwood Experiments and Simulations
}

\author{
Author: Nicholas Mueschke \\ Texas A\&M University / Lawrence Livermore National Laboratory \\ Advisor: Malcolm J. Andrews, Ph.D. \\ Texas A\&M University, Department of Mechanical Engineering \\ LLNL Supervisor: Oleg Schilling, Ph.D. \\ AX-Division, A-Program Complex Hydrodynamics Group
}

Last Revision: 7/14/03

\section{Outline of Work to be Performed}

The primary goal of the research being conducted this summer is to investigate the role of initial conditions in the development of a two fluid mix driven by RayleighTaylor instability. The effects of initial conditions will be studied through the use of experimental facilities located at the Buoyancy-Driven Mixing Lab at Texas A\&M University and through high resolution direct numerical simulations of the experiment by the MIRANDA code developed at Lawrence Livermore National Lab.

Experimental Objectives:

1. Analyze the early time development of a two fluid Rayleigh-Taylor driven mix between two miscible fluids at low Atwood numbers.

2. Quantify the initial conditions of the unstably stratified fluids by means of statistical mixing parameters and spectral analysis of the centerline density fluctuations.

3. Capture PLIF images of initial development of the flow for use in simulation setup. (Wayne Kraft)

4. Determine exactly what component of the experimental mixing data (position downstream from the splitter plate) most accurately represents the initial conditions of the experiment.

Simulation Objectives:

1. Perform two dimensional and three dimensional simulations of the experimental setup. Analyze the results of these simulations for comparison to the experimental results.

2. Various methods of implementing the initial conditions in the simulations are to be investigated. Some of those methods are:

a. Various simplified density profile assumptions will also be investigated, such as repeating saw-teeth patterns, etc. There is also a concern to add 
some degree of randomness to these simplified perturbation profile assumptions.

b. Convert portions of raw PLIF data to a set of parameterized surfaces that can be directly input as both two dimensional and three dimensional surfaces.

c. Determine and implement a method for directly converting the initial density spectral data into a density profile that can be implemented in two and three dimensional simulations.

3. Quantify the dynamical quantities associated with the evolution equations of density, kinetic energy, and enstrophy.

Modeling Objectives:

1. Perform a similar set of simulations using the artificial diffusion equations proposed by Oleg Schilling to validate their use. Results are to be compared to the experimental and DNS simulations.

2. Perform comparisons between DNS simulations of experiment and the proposed EZTurbMix models under development by Oleg Schilling.

\section{Experiments Performed}

Experiments were performed using a water channel at Texas A\&M University to investigate the effects of initial conditions on the growth of a two fluid turbulent mix induced by Rayleigh-Taylor instability. ${ }^{1,2,3}$ A water channel in which a volume of cold and warm water $\left(\Delta \mathrm{T} \approx 5^{\circ} \mathrm{C}\right)$ flow into a mixing section was used to create an unstable stratification of a dense fluid above a lighter fluid. The temperature difference in the fluids provides the difference in densities by means of thermal expansion. The volumes of cold and warm water are initially separated by a thin splitter plate as they flow towards the mixing section. The end of the splitter plate is machined to a $2.5^{\circ} \mathrm{knife}$-edge and is followed immediately by a wire mesh screen ( 35 wires-per-inch). The wire mesh screen serves the double purpose of minimizing the development of a boundary layer on the surface of the splitter plate as well as inducing small initial perturbations on the interface between the cold and warm fluids. As the two fluids flow through the channel in time, the mixing layer develops and grows in height. By means of the Taylor hypothesis, distance downstream from the splitter plate can be converted to time by $t=x / U$, where $t$ is time in seconds, $\mathrm{x}$ is position downstream from the splitter plate in $\mathrm{cm}$, and $\mathrm{U}$ is the mean flow velocity in the $\mathrm{x}$ direction in $\mathrm{cm} / \mathrm{s}$.

\subsection{Thermocouple Experiments}

Density measurements were made using an E-type thermocouple with a weld bead $0.15 \mathrm{~mm}$ in diameter. Temperature measurements were recorded at a rate of 50,000 $\mathrm{Hz}$ and then averaged over a 10 point window to eliminate noise. The effect of the timeaveraging reduced the sampling rate to an effective $5000 \mathrm{~Hz}$. The data was then converted from temperature measurements to density measurements by an equation of 
state. ${ }^{4}$ From this density data, measurements of density fluctuations, density spectra, and mixing parameters were calculated. Refer to Figure 1 for an image of approximately the first $30 \mathrm{~cm}$ of the mixing layer with the thermocouple probe located at the centerline of the mixture, $10 \mathrm{~cm}$ from the end of the splitter plate.
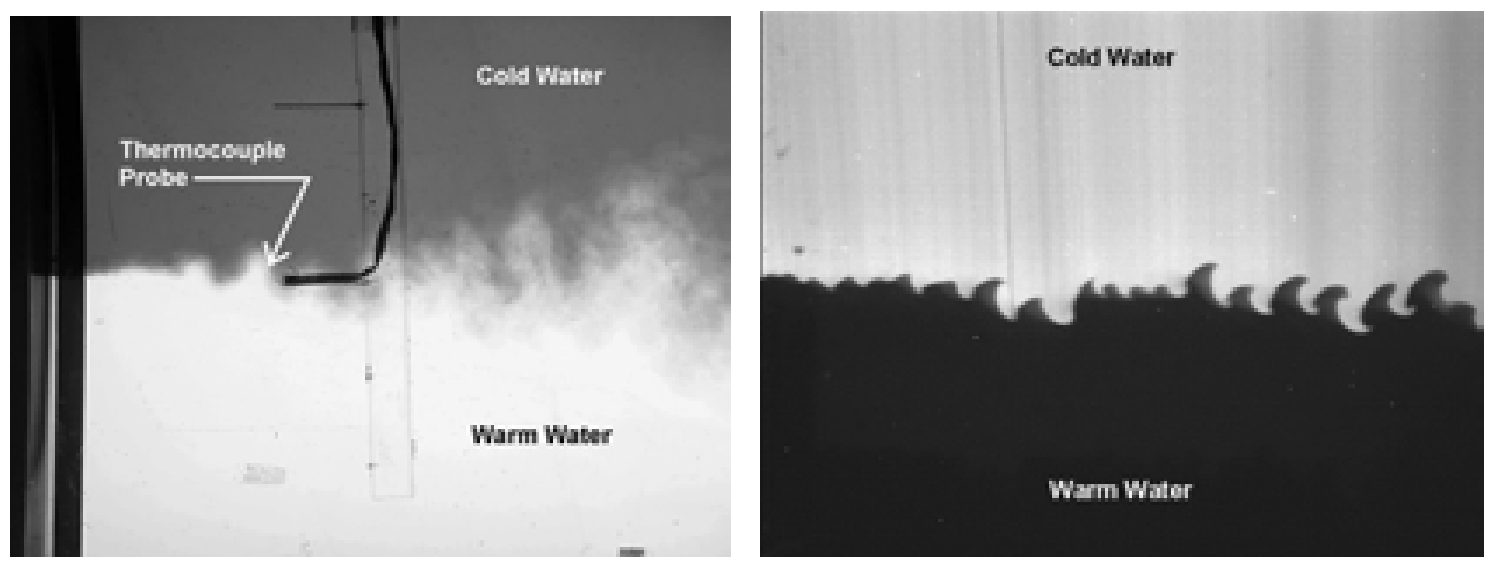

Figure 1. Picture of mixing layer (left) developing as cold water (top fluid containing a dye) and warm water (bottom fluid) come into contact after the end of the splitter plate. The mean flow velocity is from left to right. A thermocouple probe was placed in the mixing layer to make instantaneous density measurements. The tip of the thermocouple is located off the left edge of the bottom protrusion. PLIF image (right) of initial mixing layer development. The mean flow of the fluid is from left to right, where the top fluid contains a fluorescent dye that is illuminated by a laser sheet.

Data was measured at four locations downstream from the splitter plate, which can be related to four different moments in time. Density measurements were made at $1 \mathrm{~mm}, 1 \mathrm{~cm}, 5 \mathrm{~cm}$, and $10 \mathrm{~cm}$ downstream. The downstream locations can be equated to dimensional time by the Taylor hypothesis and then normalized to form a nondimensional time of the form:

$$
\tau=t\left(\frac{H}{A_{t} g}\right)^{\frac{1}{2}}=\frac{x}{U}\left(\frac{H}{A_{t} g}\right)^{\frac{1}{2}}
$$

where $t$ is time, $A_{t}$ is the Atwood number, $g$ is gravity and $H$ is the nominal height associated with a particular configuration. For the case of the water channel, $\mathrm{H}=30 \mathrm{~cm}$, $\mathrm{g}=981 \mathrm{~cm} / \mathrm{s}^{2}, \mathrm{U} \approx 4.3 \mathrm{~cm} / \mathrm{s}$. The first set of data (four downstream locations) was taken with the water channel in an "as is" state to provide a baseline set of experimental data. A second set of data was recorded in an attempt to alter the initial density fluctuation spectrum. This was accomplished by reconfiguring the cold water inlet plenum so that oscillations in the outlet of the cold water pipe would be further damped. Table 1 lists a summary of the experiments performed. 


\begin{tabular}{|c|c|c|c|c|}
\hline \multicolumn{5}{|c|}{ Summary of Experiments } \\
\hline \multicolumn{5}{|c|}{ Baseline Experiments } \\
\hline$\#$ & Downstream & $\tau$ & At & Diagnostic \\
\hline 1 & $0.1 \mathrm{~cm}$ & 0.0038 & $7.71 \mathrm{E}-04$ & Thermocouple \\
\hline 2 & $1.0 \mathrm{~cm}$ & 0.0377 & $7.66 \mathrm{E}-04$ & Thermocouple \\
\hline 3 & $5.0 \mathrm{~cm}$ & 0.1890 & $7.71 \mathrm{E}-04$ & Thermocouple \\
\hline 4 & $10.0 \mathrm{~cm}$ & 0.3800 & $7.80 \mathrm{E}-04$ & Thermocouple \\
\hline \multicolumn{5}{|c|}{ Modified Experiments } \\
\hline \multicolumn{5}{|c|}{ At } \\
\hline$\#$ & Downstream & \multicolumn{5}{|c|}{ Diagnostic } \\
\hline 1 & $0.1 \mathrm{~cm}$ & 0.0036 & $7.82 \mathrm{E}-04$ & Thermocouple \\
\hline 2 & $1.0 \mathrm{~cm}$ & 0.0362 & $7.77 \mathrm{E}-04$ & Thermocouple \\
\hline 3 & $5.0 \mathrm{~cm}$ & 0.1752 & $7.27 \mathrm{E}-04$ & Thermocouple \\
\hline 4 & $10.0 \mathrm{~cm}$ & 0.3551 & $7.47 \mathrm{E}-04$ & Thermocouple \\
\hline
\end{tabular}

Table 1. Summary of experiments performed.

\subsection{Experimental Results}

From the temperature time series, which is converted to a density time series through an equation of state, it is possible to calculate spectral information relevant to the fluctuating component of the density time series. Spectral information was calculated at all four downstream positions for both the baseline case and the modified case. Figure 2 shows the spectra for the four locations of the baseline experiment and Figure 3 shows the spectra for the modified experiments. All spectra are plotted in wavenumber space where $\mathrm{k}$ is the wavenumber and it is calculated by:

$$
k=\frac{f}{U}=\frac{1}{\lambda},
$$

where $f$ is the frequency of fluctuating density oscillations and $U$ is the mean convective velocity. The wavenumber is equivalent to the inverse of the wavelength of the oscillation being measured.

It can be seen in the both the baseline and modified experimental spectra that an inertial subrange develops early in the flow by $\tau \approx 0.18$. [Check $-5 / 3$ slope, etc.] The interesting difference between the two experiments can be seen in the earliest spectra, $\tau \approx$ 0.004 , where there is a significant drop off in density fluctuations in the low wavenumber regime. This reduction is later quantified by the mixing parameter calculations. 

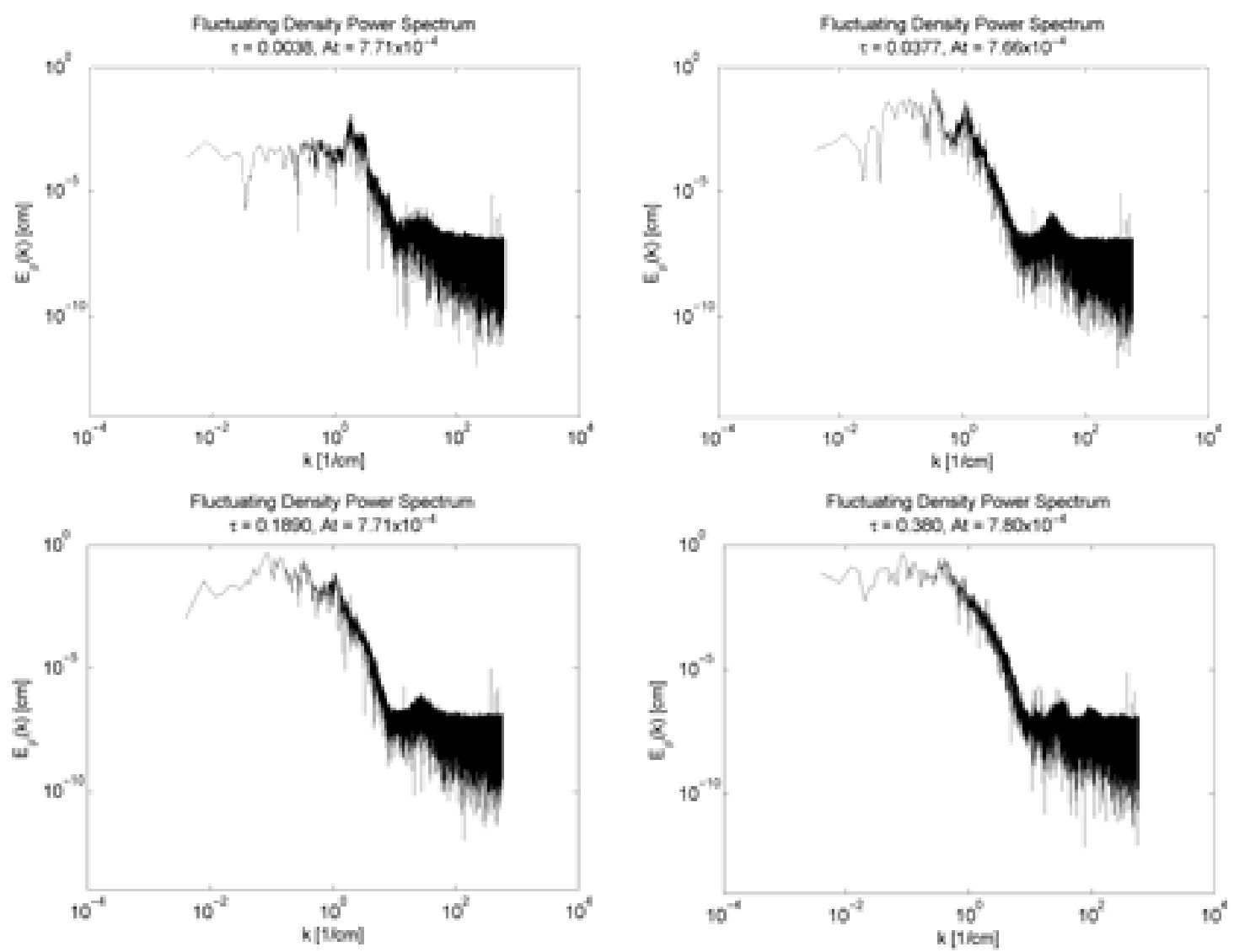

Figure 2. Plots of fluctuating density spectra in wavenumber space for the baseline experimental data.

With the density measurements recorded, it is useful to calculate the following mixing parameters ${ }^{4}$ :

$$
\begin{aligned}
& B_{o}=\lim _{T \rightarrow \infty} \frac{1}{T} \int_{0}^{T} \frac{(\rho-\bar{\rho})^{2}}{(\Delta \rho)^{2}} d t \\
& B_{2}=f_{1} f_{2}=f_{1}\left(1-f_{1}\right)=\overline{\rho^{*}}\left(1-\overline{\rho^{*}}\right), \\
& \theta=1-\frac{B_{0}}{B_{2}}
\end{aligned}
$$

In Equations 3a and 3b, $\Delta \rho=\rho_{1}-\rho_{2}$ and $\rho^{*}$ is a nondimensional density that is equivalent to the volume fraction of fluid 1 and is defined:

$$
\rho^{*}=\frac{\rho-\rho_{2}}{\rho_{1}-\rho_{2}}
$$



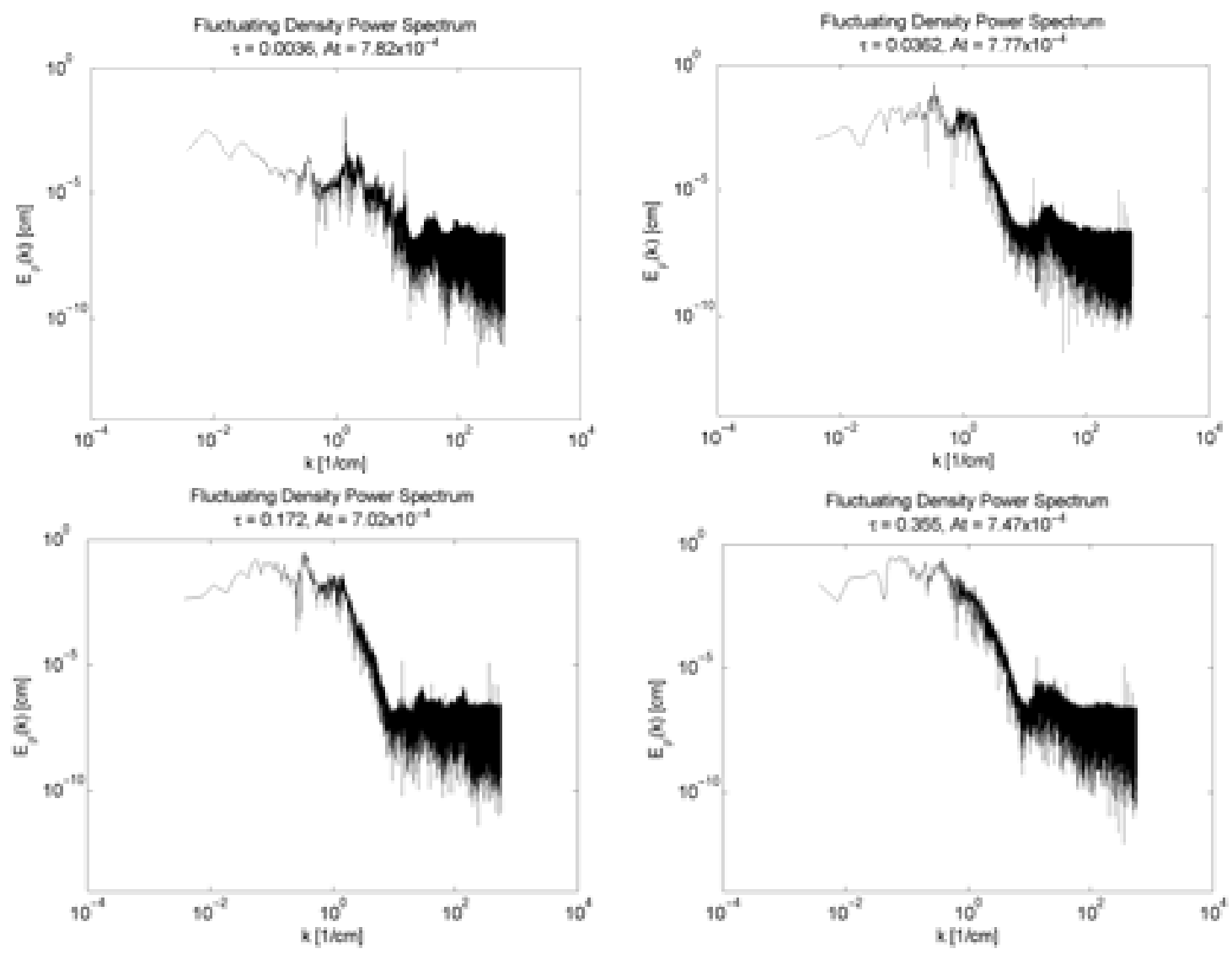

Figure 3. Plots of fluctuating density spectra in wavenumber space for the modified experimental data. Nondimensional time locations are $\tau=0.0036$ (top left), $\tau=0.0362$ (top right), $\tau=0.1752$ (bottom left) and $\tau=0.3551$ (bottom right).

Equations 3 and 4 are measures of the turbulent density fluctuations for a miscible two fluid mixture and an immiscible two fluid mixture respectively. Equation 5 is used to determine the degree of molecular mixing between the two fluids, where a value of unity represents completely molecularly mixed fluids and a value of zero represents completely unmixed fluids. ${ }^{5,6}$ The molecular mixing fraction presented here is analogous to the parameter $\Xi$ presented by Cook and Dimotakis. ${ }^{[\text {Insert Ref] }}$ Figure 4 a shows the resulting values of $B_{0}, B_{2}$, and $\theta$ at the specified measurement locations.

Returning to the difference between the baseline and modified experimental results, it can be seen that the early time $(\tau \approx 0.004)$ density spectra have a qualitatively different shape in the low wavenumber regime. This effect is also noticed in the difference in the value of $B_{0}$ at $\tau \approx 0.004$ which can be seen in Figure $4 \mathrm{~b}$. Alternatively, the parameter $\mathrm{B}_{0}$ can be defined as the area under the power spectrum curve in wavenumber space such that:

$$
B_{0}=\int_{0}^{\infty} E_{\rho^{\prime}}(k) d k
$$


With this formulation, it can be seen that a loss of energy in the density fluctuations over a range of wavenumbers will manifest itself in lowered value of $\mathrm{B}_{0}$. However, it is still uncertain as to whether or not this reduced value of $\mathrm{B}_{0}$ plays a significant role in the development of the mixing layer at later times.
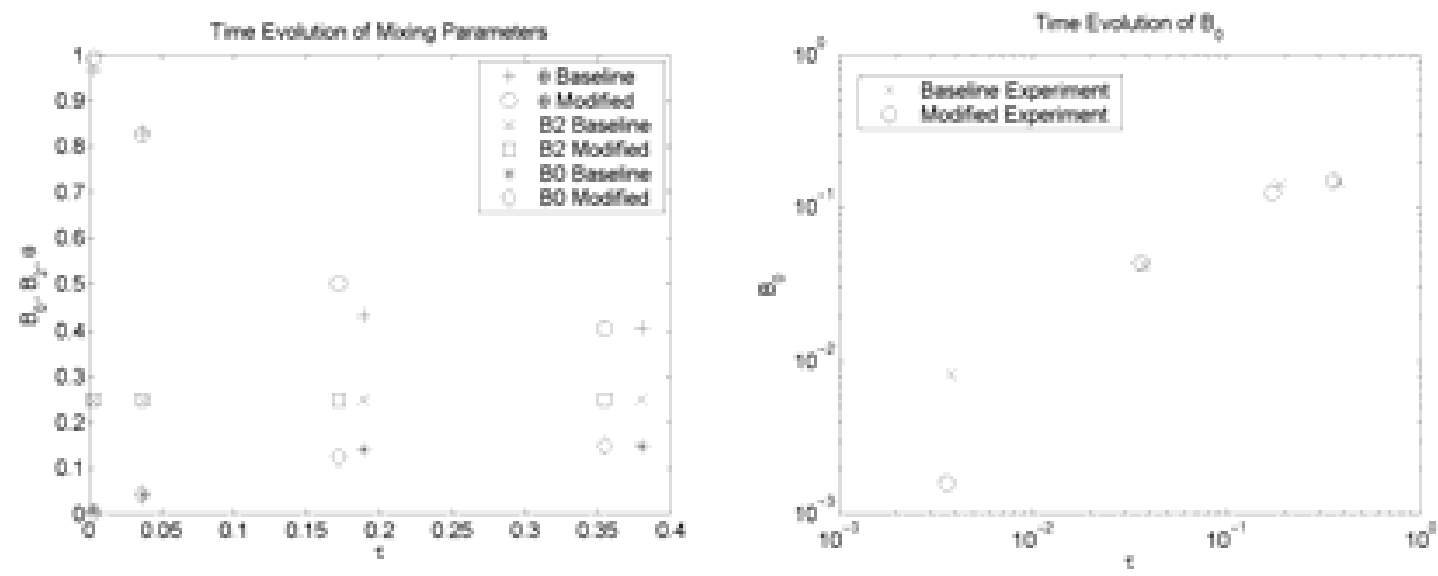

Figure 4a. (Left; Linear scale) Time evolution of mixing parameters for baseline and modified experiments.

Figure 4b. (Right; Log-Log scale) Time evolution of mixing parameter $\mathrm{B}_{0}$.

\section{Numerical Simulations Performed}

To further examine the role of initial conditions in the development of a mixing layer induced by Rayleigh-Taylor instability, direct numerical simulations (DNS) of the experiment were conducted using the MIRANDA code developed by A. W. Cook at LLNL. $^{7,8}$ All simulations are incompressible, variable density simulations. A third order, variable time step Adams-Bashforth-Moulton time-evolution scheme is employed. All spatial derivatives in the horizontal directions are computed by means of tenth-order fast Fourier transforms and tenth-order compact finite-differencing method in the vertical direction. Post-processing of the data from was done by the MIRANDA_Analysis code developed by O. Schilling at LLNL.

It is of primary interest to compare the DNS results of the simulated experiment with the experimental data to validate the both the statistical and dynamic development of the simulated mixing layer as well as to investigate the effects that the initial conditions of the flow impart to this development. To accomplish this, both statistical parameters and quantitative analysis of the evolution equations of density, kinetic energy, and enstrophy will be studied. Hopefully this will enlighten the differences between two dimensional and three dimensional simulations beyond the concept of the simple removal of vortex stretching effects.

The MIRANDA code, in DNS mode, solves the following equations: 


$$
\frac{\partial}{\partial t}\left(\begin{array}{c}
\rho \\
\rho v_{i} \\
\rho m_{\alpha}
\end{array}\right)+\frac{\partial}{\partial x_{j}}\left(\begin{array}{c}
\rho v_{j} \\
\rho v_{i} v_{j}+p \delta_{i j}-\sigma_{i j} \\
\rho m_{\alpha} v_{j}-\rho D \frac{\partial m_{\alpha}}{\partial x_{j}}
\end{array}\right)=\left(\begin{array}{c}
0 \\
\rho g_{i} \\
0
\end{array}\right)
$$

which constitute the continuity, Navier-Stokes, and mass fraction conservation equations respectively. The following notation is employed in Equation 8:

$\rho \quad$ Scalar density value, $\rho(\mathrm{x}, \mathrm{y})$,

$v_{i} \quad$ Scalar velocity magnitude in the $\mathrm{i}^{\text {th }}$ direction,

$p \quad$ Scalar pressure value,

$m_{\alpha} \quad$ Local mass fraction of fluid $\alpha$,

$g \quad$ Gravity, $g_{z}=-981 \mathrm{~cm} / \mathrm{s}^{2}$,

D Constant Fickian mass diffusivity value.

The viscous stress tensor is defined by the following:

$$
\sigma_{i j}=\mu\left(\frac{\partial v_{i}}{\partial x_{j}}+\frac{\partial v_{j}}{\partial x_{i}}-\frac{2}{3} \delta_{i j} \frac{\partial v_{k}}{\partial x_{k}}\right)
$$

where $\mu$ is the dynamic viscosity of the fluid.

For all simulations, the following values were used: $g_{z}=-981 \mathrm{~cm} / \mathrm{s}^{2}, \rho_{1}=$ $998.5986 \mathrm{~kg} / \mathrm{m}^{3}, \rho_{1}=997.0479 \mathrm{~kg} / \mathrm{m}^{3}$. The selected densities result in an Atwood number of $A_{t}=7.5 \times 10^{-4}$ and $A_{t} \times g=0.73757 \mathrm{~cm} / \mathrm{s}^{2}$.

\subsection{Initial Conditions}

In the past, extensive research has been conducted to investigate single mode and multiple mode initial density perturbations of an arbitrary choice, i.e. wave number ${ }^{9}$, initial spectrum ${ }^{7,8}$ or amplitude and phase. ${ }^{10}$ Also, some effort has been made to initialize simulations with the initial conditions from experimental data. ${ }^{11}$ There is a current interest in using statistical density data to initialize Rayleigh-Taylor simulations. The thrust of this research is to investigate alternative methods for implementing the initial conditions of the flow such that it is possible to use experimental data to directly initialize the simulation.

The initial perturbation fields for the simulations were created by the function:

$$
\rho(x, z, t=0)=\rho_{\text {avg }}+\frac{\Delta \rho}{2} \operatorname{erf}\left[\frac{z+\zeta(\mathbf{x})}{\varepsilon}\right],
$$


where $\zeta(x)$ is the dimensional perturbation field with the units of centimeters. ${ }^{7}$ In this case, $\boldsymbol{x}$ represents only the x-direction in the two dimensional simulations and both the xand $y$-directions in the three dimensional simulations. The parameter of the error function is normalized by $\varepsilon$ which has the units of centimeters and is defined by:

$$
\varepsilon=\frac{\text { Interfacial thickness }}{2}
$$

In an attempt to better represent the initial conditions of the experimental data in the simulations, two different approaches to parameterizing the initial density surface were investigated.

1. Extracting the density interface profile directly from PLIF images of the early time development of the flow.

2. Converting fluctuating density spectral information directly to a parameterized surface.

All parameterization methods were constructed through the use of a Fourier series such that the new perturbation function is of the form:

$$
\zeta(x)=\sum_{n=0}^{n_{\max }} a_{n} \cos \left(\frac{2 \pi n}{\lambda_{F}} x\right)+b_{n} \sin \left(\frac{2 \pi n}{\lambda_{F}} x\right),
$$

where $a_{n}$ and $b_{n}$ determine the shape of the repeating wave form. The variable $\lambda_{F}$ is the wavelength of the Fourier decomposed wave or profile in centimeters. For the case in which the interface profile is extracted directly from the PLIF images, the Fourier coefficients can be determined by the equations:

$$
\begin{aligned}
& a_{o}=\frac{1}{\lambda_{F}} \int_{0}^{\lambda_{F}} f_{\text {profile }}(x) d x, \\
& a_{n}=\frac{2}{\lambda_{F}} \int_{0}^{\lambda_{F}} f_{\text {profile }}(x) \cos \left(\frac{2 \pi n}{\lambda_{F}} x\right) d x, \\
& b_{n}=\frac{2}{\lambda_{F}} \int_{0}^{\lambda_{F}} f_{\text {profile }}(x) \sin \left(\frac{2 \pi n}{\lambda_{F}} x\right) d x .
\end{aligned}
$$

For the case of a two dimensions density interface perturbation function, $\zeta(x, y)$, no experimental data exists to data describing the density fluctuations in the transverse or $y$-direction, so assumptions must be made. The two dimensional perturbation function will take the form of the product of a perturbation function in the x-direction and a perturbation function in the y-direction. This will allow for the primary perturbation in the $\mathrm{x}$-direction to dominate and allow for variation of transverse perturbations to be investigated. The resulting perturbation function is as follows: 


$$
\begin{aligned}
\zeta(x, y)= & {\left[\sum_{n=0}^{N_{\max , x}} a_{n} \cos \left(\frac{2 \pi n}{\lambda_{F, x}} x\right)+b_{n} \sin \left(\frac{2 \pi n}{\lambda_{F, x}} x\right)\right] } \\
& \cdot\left[\sum_{n=0}^{N_{\max , y}} c_{n} \cos \left(\frac{2 \pi n}{\lambda_{F, y}} y\right)+d_{n} \sin \left(\frac{2 \pi n}{\lambda_{F, y}} y\right)\right] .
\end{aligned}
$$

The variables $x$ and $y$ are defined:

$$
\begin{aligned}
& x(i)=\left(\Delta x \cdot i-\frac{\Delta x}{2}\right)+\text { Offset }_{x}, \\
& y(j)=\left(\Delta y \cdot j-\frac{\Delta y}{2}\right)+\text { Offset }_{y},
\end{aligned}
$$

where $i$ and $j$ are grid point locations. The need for an Offset variable will be discussed in Section 3.3.

For the PLIF-based initial conditions sets (parameterization method \#1), several PLIF images were taken and cropped so that only the first 1-3 centimeters of the flow remained. Then a Canny type edge detection algorithm was used to determine the shape of the interface. This profile was then decomposed to a Fourier series so that the surface could be parameterized and input into a variety of simulation setups. A sample of a cropped PLIF image and resulting interface profile image are shown in Figure 5.
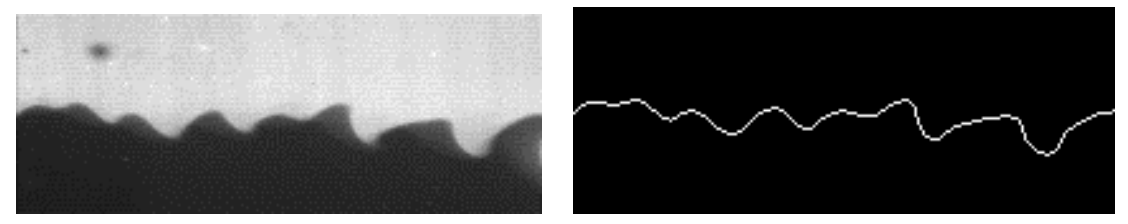

Figure 5. (Left Image) PLIF image taken directly off of the edge of the splitter plate where the initial instability develops. The image captures approximately the first $3 \mathrm{~cm}$ of the flow. (Right Image) This is the resulting image after a Canny type edge filter was used to detect the exact location of the density interface.

It was found that parameterized surfaces with $\lambda_{F}<L_{x}$ impose a periodicity on the flow that restricts the growth of the mixing layer when the mixing layer height is on the order of $\lambda_{F}$. To counter this effect, a series of randomly chosen profile images were pieced together with other PLIF images that have been randomly mirrored about either a vertical or a horizontal axis. These combined PLIF-based profiles increased the "randomness" of the initial conditions which can be qualitatively seen in the images of the density field at both early and late times.

For the case of spectral-based initial conditions (parameterization method \#2), the following method was used to calculate the $a_{n}$ and $b_{n}$ coefficients:

1. Import raw temperature data.

2. Time average the temperature data and crop it as desired.

3. Convert the temperature data to density data. 
4. Normalize the density data by dividing by $\Delta \rho$ and subtracting out the mean to get $\rho^{\prime} / \Delta \rho$.

5. Transform the data to wavenumber space by means of the FFT algorithm.

6. The resulting set of complex numbers from the Fourier transform become the $a_{n}$ and $b_{n}$ coefficients such that:

$$
\begin{aligned}
& a_{n}=\operatorname{Re}\left(F F T\left(\frac{\rho^{\prime}}{\Delta \rho}\right)\right) \\
& b_{n}=\operatorname{Im}\left(F F T\left(\frac{\rho^{\prime}}{\Delta \rho}\right)\right)
\end{aligned}
$$

7. All wavenumbers above a specified limit are cropped so to eliminate any effects due to thermocouple noise and to reduce the size of the input file to a manageable size.

8. The $a_{n}$ and $b_{n}$ coefficients are then scaled so that the RMS value of the perturbation function $\zeta$ matches a similar value RMS value for the PLIF-based perturbation functions. $\left(\zeta_{\mathrm{rms}, 0.1 \mathrm{~cm}}=0.05 ?, \zeta_{\mathrm{rms}, 1.0 \mathrm{~cm}}=0.15\right.$ ? $)$

Refer to Figure 6 for plot of the $\left|a_{n}\right|$ and $\left|b_{n}\right|$ coefficients.
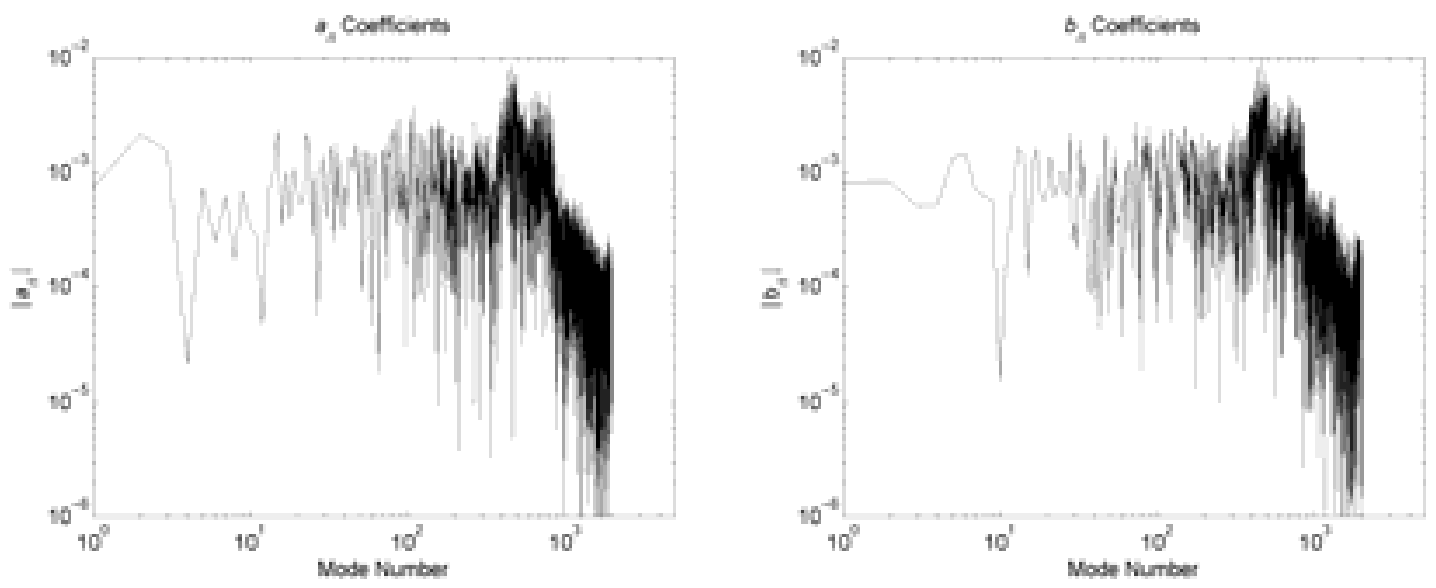

Figure 6. Scaled and cropped Fourier coefficients from $0.1 \mathrm{~cm}$ baseline density data.

\subsection{Two Dimensional Simulations}

Two dimensional simulations are either of the size $\left(\mathrm{L}_{\mathrm{x}} \times \mathrm{L}_{\mathrm{z}}\right) 16 \mathrm{~cm} \times 16 \mathrm{~cm}$ or $32 \mathrm{~cm} \times 32 \mathrm{~cm}$, where both were run at a resolution $\left(\mathrm{N}_{\mathrm{x}} \times \mathrm{N}_{\mathrm{z}}\right)$ of $1024 \times 1024$ grid points. Figures 8 and 9 show the time evolution of two dimensional mixing layer initialized with PLIF-based initial conditions and spectral-based initial conditions, respectively. 

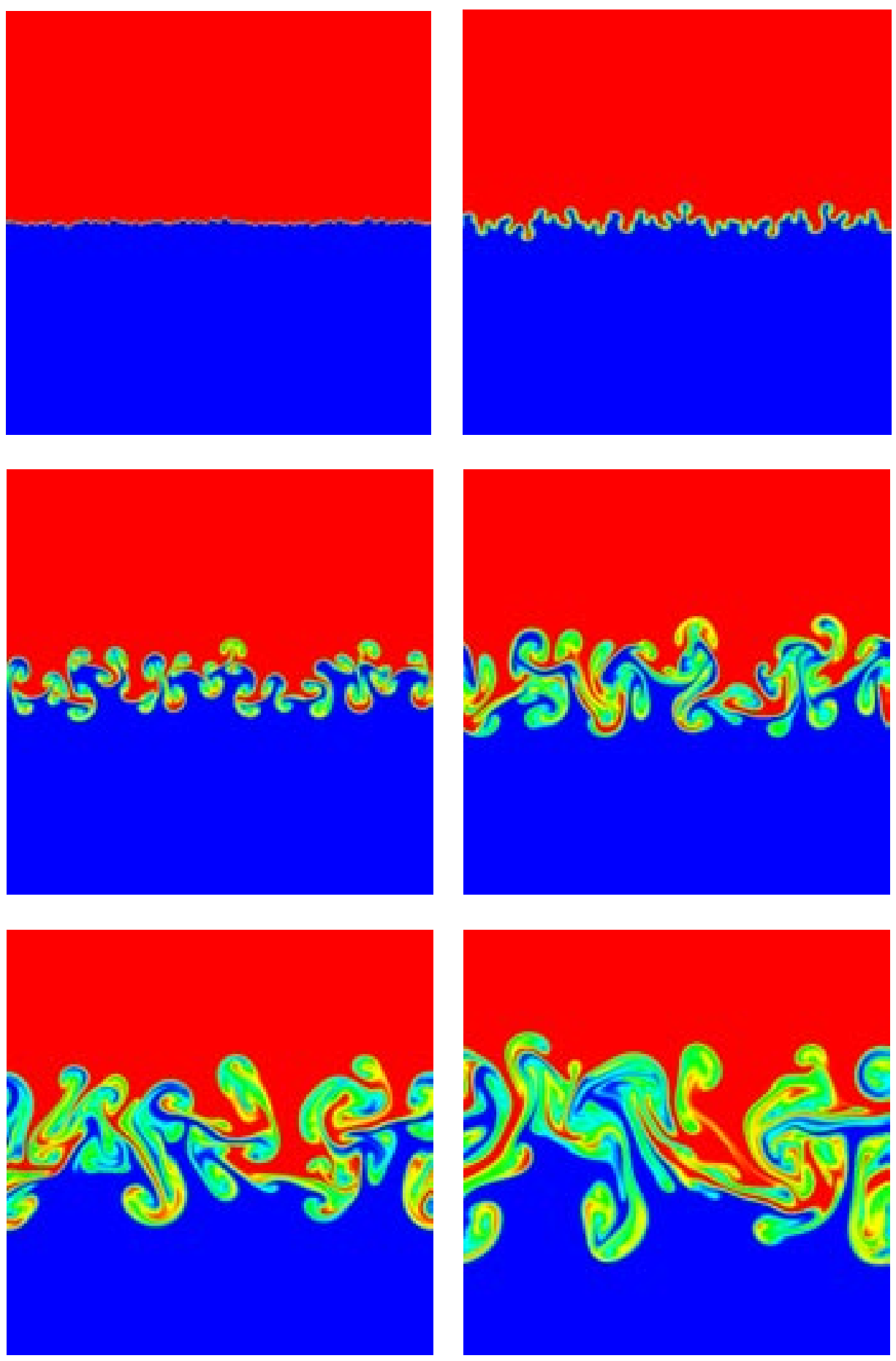

Figure 7. Two dimensional simulation of Rayleigh-Taylor mixing layer using PLIFbased initial conditions (parameterization method $\# 1$ ). Images are shown at $\mathrm{t}=0$ (top left), $\mathrm{t}=2$ (top right), $\mathrm{t}=4$ (middle left), $\mathrm{t}=6$ (middle right), $\mathrm{t}=8$ (bottom left), and $\mathrm{t}=$ 10 (bottom right). The simulation was run on ASCI Blue with a grid size of $1024 \times 1024$ $[16 \mathrm{~cm} \times 16 \mathrm{~cm}]$. 

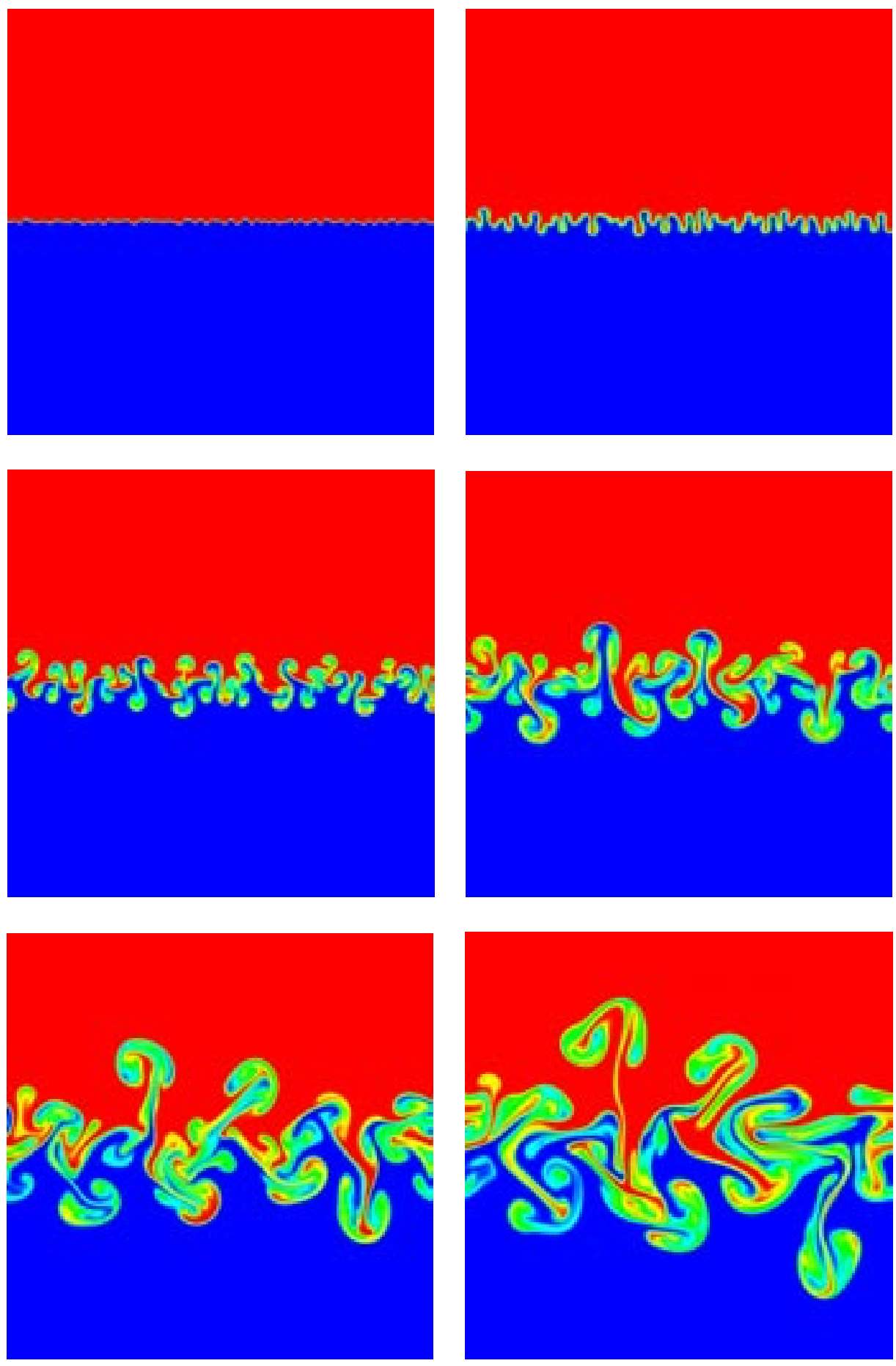

Figure 8. Two dimensional simulation of Rayleigh-Taylor mixing layer using spectralbased initial conditions (parameterization method \#2). Images are shown at $\mathrm{t}=0$ (top left), $\mathrm{t}=2$ (top right), $\mathrm{t}=4$ (middle left), $\mathrm{t}=6$ (middle right), $\mathrm{t}=8$ (bottom left), and $\mathrm{t}=$ 10 (bottom right). The simulation was run on ASCI Blue with a grid size of $1024 \times 1024$ $[16 \mathrm{~cm} \times 16 \mathrm{~cm}]$. 
It was observed in the two dimensional spectral-based simulations that different sections of the flow evolve at slightly different growth rates, presumably due to the local initial conditions. Figure 9 shows a section of a $32 \mathrm{~cm} \times 32 \mathrm{~cm}(1024 \times 1024)$ simulation in which the same spectral information was used to initialize the flow as used in the $16 \mathrm{~cm} \times 16 \mathrm{~cm}$ simulation depicted in Figure 8 . It can be seen that the left half of the image is nearly identical to the $16 \mathrm{~cm} \times 16 \mathrm{~cm}$ simulation at $\mathrm{t}=10$ shown in Figure 8 . This is expected because of the use of the same initial conditions. What was not expected was to see a fairly dramatic difference in the development in the mixing layer between the left $16 \mathrm{~cm}$ and the right $16 \mathrm{~cm}$ for the same set of initial conditions. The difference is attributed to the inability to convey all of the information from the spectral-based initial conditions sets to a $32 \mathrm{~cm}$ domain width. By using an Offset variable in the definition of the $x$, see Equation it is possible to translate the domain so that a separate section of the spectral initial condition can be implemented. There is an interest to try several sets of initial conditions with the same spectral-based set to compare and contrast any differences.

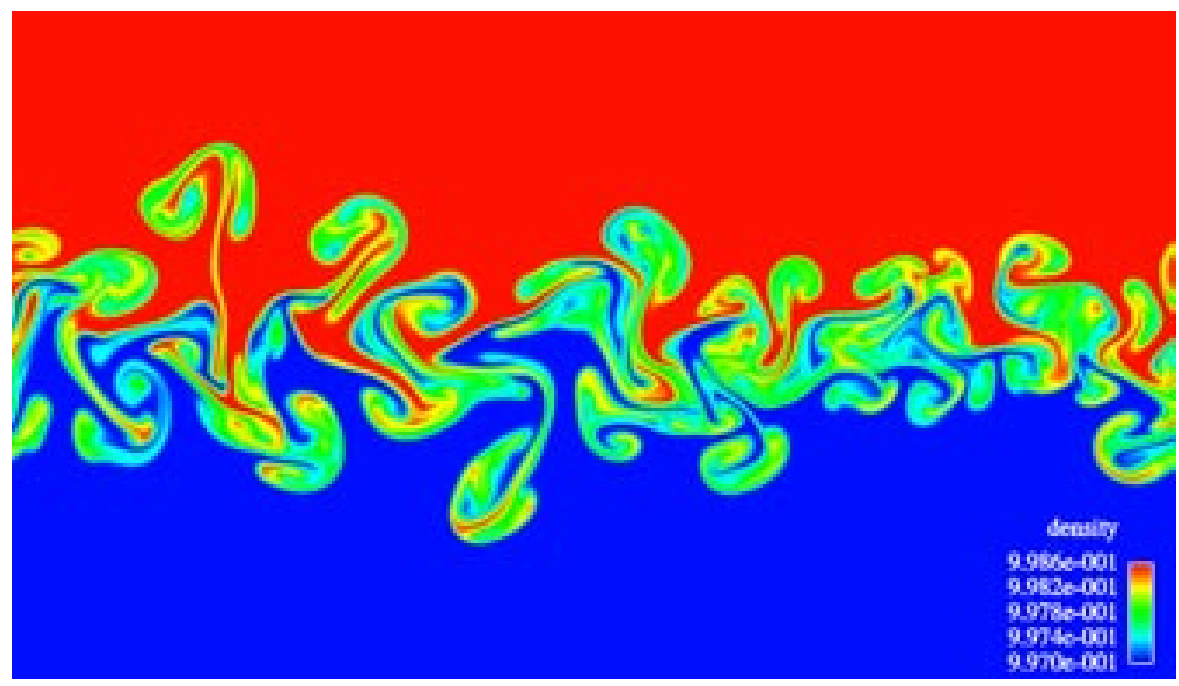

Figure 9. Two dimensional simulation of Rayleigh-Taylor mixing layer using spectralbased initial conditions shown at $\mathrm{t}=10$. The simulation was run on ASCI Blue with a grid size of $1024 \times 1024[16 \mathrm{~cm} \times 16 \mathrm{~cm}]$.

\subsection{Three Dimensional Simulations}

It has been noted in from observations made of the water channel experiment that the initial perturbations of the fluid interface are primarily a two dimensional perturbation. For this reason, it is desirable to show that a three dimensional simulation of an RT driven mixing layer will develop only the two dimensional perturbations during the linear stage before becoming a fully three dimensional developed mixing layer at the onset of self-similarity.

The three dimensional simulations run in this investigation are either of the size $\left(\mathrm{L}_{\mathrm{x}} \times \mathrm{L}_{\mathrm{y}} \times \mathrm{L}_{\mathrm{z}}\right) 16 \mathrm{~cm} \times 8 \mathrm{~cm} \times 16 \mathrm{~cm}$ or $32 \mathrm{~cm} \times 16 \mathrm{~cm} \times 32 \mathrm{~cm}$. 


\section{Comparison of Experimental and Simulation Results}

1. Growth rates, Re values, large scale qualitative results.

2. Statistical results, spectral, etc.

3. Mixing parameters, etc.

\section{Future Work}

There is an interest in extending the comparison between the experimental and simulation data to include the statistical quantities of dynamic values, such as kinetic energy and enstrophy. It is believed that more accurate comparison of the initial conditions can be achieved by the comparison of early time kinetic energy and vorticity production.

It is also of interest, for validation purposes, to compare the experimental data to statistical data and mixing parameters extracted from 'pseudo-DNS' simulations of the experiment.

\footnotetext{
${ }^{1}$ D. M. Snider, M. J. Andrews, "Rayleigh-Taylor and shear driven mixing with an unstable thermal stratification," Physics of Fluids 6, 3324-3334 (1994).

${ }^{2}$ P. N. Wilson, M. J. Andrews, F. Harlow, "Spectral nonequilibrium in a turbulent mixing layer," Physics of Fluids 11, 2425-2433 (1999).

${ }^{3}$ P. N. Wilson, M. J. Andrews, "Spectral measurements of Rayleigh-Taylor mixing at small Awood number," Physics of Fluids 14, 938-945 (2001).

${ }^{4}$ Equation of state reference.

${ }^{5}$ Youngs (1991).

${ }^{6}$ Linden et. al. (1994).

${ }^{7}$ A. W. Cook, P. E. Dimotakis, "Transition stages of Rayleigh-Taylor instability between miscible fluids," Journal of Fluid Mechanics 443, 69-99 (2001).

${ }^{8}$ A. W. Cook, Y. Zhou, "Energy Transfer in Rayleigh-Taylor instability," Physical Review E 66, 0263121-12 (2002).

${ }^{9}$ Single Mode Refs

${ }^{10}$ T. T. Clark, "A numerical study of the statistics of a two-dimensional Rayleigh-Taylor mixing layer," Physics of Fluids 15, 2413-2423 (2003).

${ }^{11}$ Youngs
} 


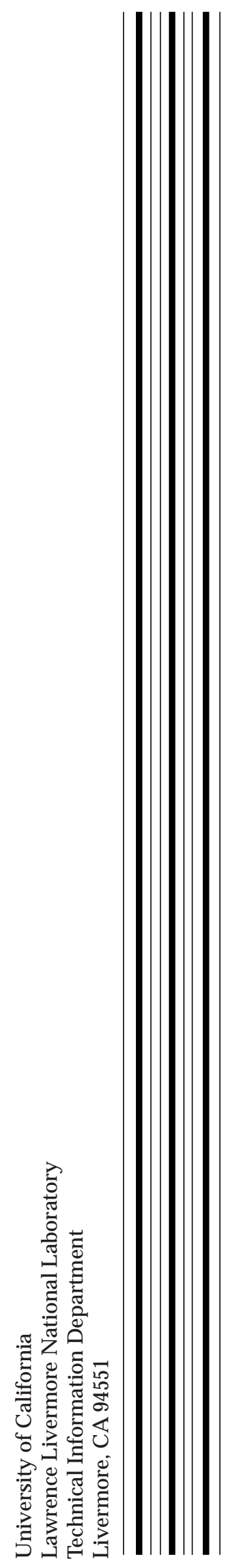

